\title{
Hopf Bifurcation of Limit Cycles in Discontinuous Liénard Systems
}

\author{
Yanqin Xiong and Maoan Han \\ Department of Mathematics, Shanghai Normal University, Shanghai 200234, China \\ Correspondence should be addressed to Maoan Han, mahan@shnu.edu.cn
}

Received 26 April 2012; Accepted 8 August 2012

Academic Editor: Alberto D’Onofrio

Copyright (c) 2012 Y. Xiong and M. Han. This is an open access article distributed under the Creative Commons Attribution License, which permits unrestricted use, distribution, and reproduction in any medium, provided the original work is properly cited.

We consider a class of discontinuous Liénard systems and study the number of limit cycles bifurcated from the origin when parameters vary. We establish a method of studying cyclicity of the system at the origin. As an application, we discuss some discontinuous Lienard systems of special form and study the cyclicity near the origin.

\section{Introduction and Main Results}

As well known, Liénard systems describe the dynamics of systems of one degree of freedom under existence of a linear restoring fore and a nonlinear dumping. In the first half of the last century models based on the Lienard system were important for the development of radio and vacuum tube technology. Nowadays the system is widely used to describe oscillatory processes arising in various studies of mathematical models of physical, biological, chemical, epidemiological, physiological, economical, and many other phenomena (see Glade et al. [1], Llibre [2] and references therein). Further, quadratic systems and some other systems can be transformed into Liénard systems by suitable changes, see for instance Han et al. [3], Cherkas [4], Gasull [5], Giné, and Llibre [6]. As we have seen, the main concern on Liénard systems is the center and focus problem and the number of limit cycles, see [7-17], and references therein. Here, we briefly list some known results related to our study in this paper. Consider a Lienard system of the form

$$
\begin{gathered}
\dot{x}=y-F(x), \\
\dot{y}=-g(x),
\end{gathered}
$$

where we suppose that the functions $F$ and $g$ satisfy two assumptions below. 
(I) There exists a positive number $\varepsilon_{0}$ such that $F$ is continuous for $|x|<\varepsilon_{0}$ with $F(0)=0$ and $g$ is continuous for $0<|x|<\varepsilon_{0}$ with $x g(x)>0$, and $\lim _{x \rightarrow 0^{+}} g(x), \lim _{x \rightarrow 0^{-}} g(x)$ existing.

(II) For $|x|<\varepsilon_{0},|F(x)|<a_{1} \sqrt{G(x)}$, where $0<a_{1}<\sqrt{8}$ and $G(x)=\int_{0}^{x} g(u) d u$. Obviously, if $g(0)=0$, system (1.1) has a singular point at the origin. By Han and Zhang [12], the origin is a generalized singular point if $g(0) \neq 0$. For example, the following system:

$$
(\dot{x}, \dot{y})= \begin{cases}(y,-1), & x \geq 0, \\ (y, 1), & x<0\end{cases}
$$

has a generalized center at the origin.

Under the condition (I), the equation $G(x)=z$ has two solutions $x=x_{1}(z)>0$ and $x=x_{2}(z)<0$ for $0<z \ll 1$. Let $F_{i}(z)=F\left(x_{i}(z)\right), i=1,2$. Then in 1952, Filippov proved the following theorem (see Chapter 5 of Ye [15]).

Theorem 1.1. Let (I) and (II) be satisfied. Further suppose $g$ in (1.1) is continuous at $x=0$. Then system (1.1) has a stable focus (resp., center, unstable focus) if

$$
F_{2}(z)-F_{1}(z)<0 \quad(\text { resp., } \equiv 0,>0) \text { for } 0<z \ll 1 .
$$

In 1985, Han [10] obtained the following.

Theorem 1.2. Consider the system

$$
\begin{gathered}
\dot{x}=h(y)-F(x), \\
\dot{y}=-g(x),
\end{gathered}
$$

where $h, F$, and $g$ are continuous functions satisfying

(i) $h(y)=($ sgny $)|y|^{\bar{m}}+O\left(y^{\bar{m}+1}\right)$ with $\bar{m}>0$,

(ii) $x g(x)>0$ for $0<|x| \ll 1$ and

$$
|F(x)|<c[G(x)]^{\bar{m} /(\bar{m}+1)} \quad \text { for }|x| \ll 1,
$$

where $0<c<(\bar{m}+1)((\bar{m}+1) / \bar{m})^{\bar{m} /(\bar{m}+1)}$. Let $\alpha(x)=-x+O\left(x^{2}\right)$ satisfy $G(\alpha(x))=G(x)$ for $|x| \ll 1$. Then system (1.4) has a stable focus (resp., center, unstable focus) if

$$
F(\alpha(x))-F(x)<0 \quad(\text { resp., } \equiv 0,>0)
$$

for $|x| \ll 1$. 
For center conditions, in 1976, Cherkas [7] proved the following.

Theorem 1.3. Let the functions $F$ and $g$ in system (1.1) be polynomials in $x$ with

$$
F(0)=g(0)=0, \quad g^{\prime}(0)>0 .
$$

Then the origin is a center of system (1.1) if and only if the equations

$$
F(x)=F(y), \quad G(x)=G(y)
$$

have a unique solution $y=\alpha(x)<0$ for $x>0$ sufficiently small.

From Cherkas [7], one can see that the above result is also true for analytic system (1.1). In 1998, Gasull and Torregrosa [9] studied the center problem for analytic systems of form (1.4) using the Cherkas' method, generalized Theorem 1.3 to (1.4) and presented interesting applications to some polynomial systems. Then in 2006, Cherkas and Romanovski [8] gave a necessary and sufficient condition for a Liénard system with nonlinearities of degree six to have a center at the origin. About Theorem 1.2, we have the following two remarks.

Remark 1.4. By the variable change

$$
u=(\operatorname{sgn} x) \sqrt{2 G(x)}
$$

and the scaling of the time $d \tau=(g(X(u)) / u) d t$, we can obtain from (1.4)

$$
\begin{gathered}
\dot{u}=h(y)-F(X(u)), \\
\dot{y}=-u,
\end{gathered}
$$

where $X(u)$ satisfies $|u|=\sqrt{2 G(X(u))}, u X(u)>0$. Thus, Theorem 1.2 is also true if $g(x)$ is not continuous at $x=0$.

Remark 1.5. If $F$ and $g$ are $C^{\infty}$ with $g^{\prime}(0)>0$. Then $F(\alpha(x))-F(x)$ in (1.6) has the form

$$
F(\alpha(x))-F(x)=\sum_{j \geq 1} B_{j} x^{j}
$$

In this case, Han [11] proved that if $B_{j}=0$ for $j=1, \ldots, 2 k-1(k \geq 1)$, then $B_{2 k}=0$, and the origin is a focus of order $k$ if $B_{2 k+1} \neq 0$ in addition. Further, if a vector parameter $a$ appears in $F$, then $B_{j}=B_{j}(a)$ and

$$
B_{2 k}=O\left(\left|B_{1}, B_{3}, \ldots, B_{2 k-1}\right|\right)
$$

for all $a$ and any $k \geq 1$. 
In this paper, we consider the following discontinuous Liénard system:

$$
\begin{gathered}
\dot{x}=h(y)-F(x, a), \\
\dot{y}=-g(x, b),
\end{gathered}
$$

where $(a, b) \in R^{n_{1}}$ with $n_{1}$ being an integer, $h(y)$ is a $C^{\infty}$ function satisfying $h(y)=y+O\left(y^{2}\right)$ and

$$
F(x, a)=\left\{\begin{array}{ll}
F^{+}(x, a), & x \geq 0, \\
F^{-}(x, a), & x<0,
\end{array} \quad g(x)= \begin{cases}g^{+}(x, b), & x \geq 0 \\
g^{-}(x, b), & x<0\end{cases}\right.
$$

where $F^{+}, g^{+}$and $F^{-}, g^{-}$are $C^{\infty}$ on $\left[0, x_{0}\right]$, and $\left[-x_{0}, 0\right]$, respectively, with $x_{0}>0$. Further, suppose there exist integers $m \geq 1, n \geq 1, k \geq 0, l \geq 0$ such that

$$
F^{+}(x, a)=\sum_{j \geq m} F_{j}^{+}(a) x^{j}, \quad g^{+}(x, b)=\sum_{j \geq k} g_{j}^{+}(b) x^{j}, \quad g_{k}^{+}(b)>0
$$

for $0<x \ll 1$ and

$$
F^{-}(x, a)=\sum_{j \geq n} F_{j}^{-}(a) x^{j}, \quad g^{-}(x, b)=g_{l}^{-}(b)(-x)^{l}+\sum_{j \geq l+1} g_{j}^{-}(b) x^{j}, \quad g_{l}^{-}(b)<0
$$

for $0<-x \ll 1$.

Let

$$
G(x, b)=\int_{0}^{x} g(u, b) d u= \begin{cases}G^{+}(x, b), & 0 \leq x \leq x_{0} \\ G^{-}(x, b), & 0<-x \leq x_{0}\end{cases}
$$

where

$$
\begin{gathered}
G^{+}(x, b)=\frac{g_{k}^{+}(b)}{k+1} x^{k+1}+\sum_{j \geq k+1} \frac{g_{j}^{+}(b)}{j+1} x^{j+1} \\
G^{-}(x, b)=\frac{-g_{l}^{-}(b)}{l+1}(-x)^{l+1}+\sum_{j \geq l+1} \frac{g_{j}^{-}(b)}{j+1} x^{j+1}
\end{gathered}
$$

for $0<x \ll 1$ and $0<-x \ll 1$, respectively.

This paper is devoted to studying the local property of system (1.13) at the origin and the number of limit cycles bifurcated from the origin as $(a, b)$ varies. The authors [13] considered system (1.13) for the case $m=n=k=l=1$ and studied the center focus problem. 
It is easy to prove that for system (1.13) under (1.15) and (1.16), the condition (ii) of Theorem 1.2 is equivalent to the following:

$$
\begin{array}{ll}
\left(\mathrm{H}_{1}\right) m>((k+1) / 2) \text { or } m=((k+1) / 2), & \left|F_{m}^{+}(a)\right|<c\left(\left(g_{k}^{+}(b)\right) /(k+1)\right)^{1 / 2}, \\
\left(\mathrm{H}_{2}\right) n>((l+1) / 2) \text { or } n=((l+1) / 2), & \left|F_{n}^{-}(a)\right|<c\left(\left(-g_{l}^{-}(b)\right) /(l+1)\right)^{1 / 2},
\end{array}
$$

where $0<c<2 \sqrt{2}$. For convenience, introduce

$$
r=\frac{k+1}{l+1}=\frac{q}{p}
$$

where $p, q$ are relatively prime numbers, that is, $(p, q)=1$. Then it is easy to see that there must exist an integer $\eta \in[0, p-1]$ such that $(n+\eta) r$ is an integer, and our main results are the following.

Theorem 1.6. Let (1.15), (1.16), $\left(H_{1}\right)$, and $\left(H_{2}\right)$ hold. Then

(i) $F^{-}(\alpha(x), a)-F^{+}(x, a)$ can be expressed as

$$
F^{-}(\alpha(x, b), a)-F^{+}(x, a)=\sum_{j \geq 1} B_{j}(a, b) x^{k_{j}},
$$

where $\alpha(x, b)=-\left[\left((l+1) g_{k}^{+}(b)\right) /\left(-(k+1) g_{l}^{-}(b)\right)\right]^{1 /(l+1)} x^{r}(1+o(1))$ satisfies $G^{-}(\alpha(x, b)$, $b)=G^{+}(x, b)$ for $x>0$ small and $k_{\tau} \in\{(n+i) r+j \mid 0 \leq i \leq p-1, i \neq \eta, j \geq 0\} \cup\{j \mid j \geq$ $\min \{m,(n+\eta) r\}\}$ for $\tau \geq 1$ with $\min \{n r, m\}=k_{1}<k_{2}<\cdots$.

(ii) If there exist $\left(a_{0}, b_{0}\right) \in R^{n_{1}}$ and $s(\geq 1)$ such that $B_{s+1}\left(a_{0}, b_{0}\right) \neq 0$ and

$$
B_{j}\left(a_{0}, b_{0}\right)=0, \quad j=1, \ldots, s, \quad \operatorname{rank} \frac{\partial\left(B_{1}, B_{2}, \ldots, B_{s}\right)}{\partial(a, b)}\left(a_{0}, b_{0}\right)=s,
$$

where $n_{1} \geq s$, then system (1.13) has cyclicity s near the origin for all $(a, b)$ near $\left(a_{0}, b_{0}\right)$.

Theorem 1.7. Let (1.15), (1.16), $\left(H_{1}\right)$, and $\left(H_{2}\right)$ hold. If there exists $s \geq 1$ such that

$$
F^{-}(\alpha(x, b), a) \equiv F^{+}(x, a) \quad \text { as } B_{1}=B_{2}=\cdots=B_{s}=0
$$

for $x \geq 0$ small and (1.22) is satisfied, then system (1.13) has cyclicity s-1 at the origin for $\left|a-a_{0}\right|+$ $\left|b-b_{0}\right|$ small.

In many cases, the function $F$ in (1.13) is linear in $a$. Then the coefficients $B_{j}(a, b)(j \geq$ $1)$ in (1.21) are linear in $a$. Let there exist $a_{0} \in R^{n_{2}}, b_{0} \in R^{n_{3}}\left(n_{2}+n_{3}=n_{1}\right)$ and integer $s>0$ $\left(s \leq n_{2}\right)$ such that

$$
B_{j}\left(a_{0}, b_{0}\right)=0, \quad j=1,2, \ldots, s, \quad \operatorname{det} \frac{\partial\left(B_{1}, B_{3}, \ldots, B_{s}\right)}{\partial\left(a_{1}, a_{2}, \ldots, a_{s}\right)}\left(a_{0}, b_{0}\right) \neq 0
$$


which implies that the linear equations $B_{j}=0, j=1,2, \ldots, s$ of $a$ have a unique solution of the form

$$
\left(a_{1}, \ldots, a_{s}\right)=\varphi\left(a_{s+1}, \ldots, a_{n_{2}}, b\right)
$$

for $b$ near $b_{0}$. Obviously, $\varphi$ is linear in $a_{s+1}, \ldots, a_{n_{2}}$. Further, let

$$
\left.B_{s+j}\right|_{\left(a_{1}, \ldots, a_{s}\right)=\varphi\left(a_{s+1}, \ldots, a_{n_{2}}, b\right)}=L_{j}\left(a_{s+1}, \ldots, a_{n_{2}}\right) \Delta_{j}(b), \quad j=1, \ldots, \sigma
$$

for some integer $\sigma>0$. Then, we can obtain the following.

Theorem 1.8. Consider system (1.13), where the function $F$ is linear in $a \in R^{n_{2}}$. Suppose (1.15), (1.16), $\left(H_{1}\right)$, and $\left(H_{2}\right)$ hold. Let there exist integers $s>0, \sigma>0$ and points $a_{0}=\left(a_{10}, \ldots, a_{n_{2}, 0}\right) \in R^{n_{2}}$ and $b_{0} \in R^{n_{3}}$ such that (1.24) and (1.26) hold with

$$
L_{j}\left(a_{s+1,0}, \ldots, a_{n_{2}, 0}\right) \neq 0, \quad j=1, \ldots, \sigma, \quad \Delta_{j}\left(b_{0}\right)=0, \quad j=1, \ldots, \sigma-1 .
$$

(i) If $\Delta_{\sigma}\left(b_{0}\right) \neq 0$ and

$$
\operatorname{rank} \frac{\partial\left(\Delta_{1} \ldots, \Delta_{\sigma-1}\right)}{\partial\left(b_{1}, \ldots, b_{\sigma-1}\right)}\left(b_{0}\right)=\sigma-1
$$

then system (1.13) has cyclicity $s+\sigma-1$ limit cycles near the origin for all $(a, b)$ near $\left(a_{0}, b_{0}\right)$.

(ii) If $\Delta_{\sigma}\left(b_{0}\right)=0$ and $F^{-}(\alpha(x), a) \equiv F^{+}(x, a)$ as $\left(a_{1}, \ldots, a_{s}\right)=\varphi\left(a_{s+1}, \ldots, a_{n_{2}}, b\right), \Delta_{j}(b)=0$, $j=1, \ldots, \sigma$ with

$$
\operatorname{rank} \frac{\partial\left(\Delta_{1}, \ldots, \Delta_{\sigma}\right)}{\partial\left(b_{1}, \ldots, b_{\sigma}\right)}\left(b_{0}\right)=\sigma
$$

then system (1.13) has cyclicity $s+\sigma-1$ limit cycles at the origin for all $(a, b)$ near $\left(a_{0}, b_{0}\right)$.

The conclusion (i) of Theorem 1.8 can be proved in a similar manner to the proof of Theorem 2 of [16] and (ii) can be verified by Theorem 1.7. Thus, we do not verify it here. The proof of Theorems 1.6 and 1.7 is presented in Section 2. In Section 3, we give some applications.

\section{Proof of Theorems 1.6 and 1.7}

In this section, we verify Theorems 1.6 and 1.7. Before proving them, we need to introduce a bifurcation function $d$ of system (1.13) and establish some preliminary lemmas, which will be used in our proof. First, we have the following lemma. 
Lemma 2.1. Suppose (1.18) holds. Then the function $\alpha(x, b)$ defined in Theorem 1.6 has the form

$$
\alpha(x, b)=\sum_{i=1}^{p} \sum_{j \geq 0} \alpha_{i j} x^{i r+j}
$$

for $0<x \ll 1$ with $\alpha_{10}=-\left[\left((l+1) g_{k}^{+}(b)\right) /\left(-(k+1) g_{l}^{-}(b)\right)\right]^{1 /(l+1)}$.

Proof. Consider the equation

$$
G^{-}(u, b)=G^{+}(x, b)
$$

for $0<-u \ll 1$ and $0<x \ll 1$. By (1.18), we can obtain

$$
\begin{aligned}
{\left[G^{-}(u, b)\right]^{1 /(l+1)} } & =\left(\frac{-g_{l}^{-}(b)}{l+1}\right)^{1 /(l+1)}(-u)\left[1+\sum_{j \geq l+1} \frac{(l+1) g_{j}^{-}(b)}{(-1)^{l+2}(j+1) g_{l}^{-}(b)} u^{j-l}\right]^{1 /(l+1)}, \\
{\left[G^{+}(x, b)\right]^{1 /(l+1)} } & =\left(\frac{g_{k}^{+}(b)}{k+1}\right)^{1 /(l+1)} x^{(k+1) /(l+1)}\left[1+\sum_{j \geq k+1} \frac{(k+1) g_{j}^{+}(b)}{(j+1) g_{k}^{+}(b)} x^{j-k}\right]^{1 /(l+1)} \\
& =\left(\frac{g_{k}^{+}(b)}{k+1}\right)^{1 /(l+1)} x^{(k+1) /(l+1)} \sum_{j \geq 0} \tilde{v}_{j} x^{j}=\sum_{j \geq 0} v_{j} x^{j+r} \equiv v(x),
\end{aligned}
$$

where $v_{0}=\left(\left(g_{k}^{+}(b)\right) /(k+1)\right)^{1 /(l+1)}$. The implicit function theorem implies that the equation $\left[G^{-}(u, b)\right]^{1 /(l+1)}=v$ has a unique solution

$$
u=u^{*}(v)=\sum_{i \geq 1} u_{i} v^{i}
$$

for $v>0$ small with $u_{1}=\left((l+1) /\left(-g_{l}^{-}(b)\right)\right)^{1 /(l+1)}$. Let $\alpha(x, b)=u^{*}(v(x))$. Then, $u=\alpha(x, b)$ for $x>0$ sufficiently small is the solution of (2.2). Combining (2.3) and (2.4), the above formula can be represented as

$$
\begin{aligned}
\alpha(x, b) & =\sum_{i \geq 1} u_{i}\left(\sum_{j \geq 0} v_{j} x^{j+r}\right)^{i}=\sum_{i \geq 1} \sum_{j \geq 0} \tilde{\alpha}_{i j} x^{i r+j} \\
& =\sum_{i \geq 1} \sum_{j \geq 0} \tilde{\alpha}_{i j} x^{(q i / p)+j}=\sum_{i=1}^{p} \sum_{j \geq 0} \alpha_{i j} x^{i r+j}
\end{aligned}
$$

with $\alpha_{10}=v_{0} u_{1}$. Thus, the proof is ended. 
Following the idea of Han [11], we have the following lemma.

Lemma 2.2. Let (1.15) and (1.16) hold. Then system (1.13) is equivalent to

$$
\begin{gathered}
\dot{u}=v-K(v) F^{*}(u, a, b), \\
\dot{v}=-u,
\end{gathered}
$$

where $K(v)=(1 / \sqrt{2})+O(v) \in C^{\infty}$, and

$$
F^{*}(u, a, b)= \begin{cases}\sum_{j \geq m} f_{j}^{+} u^{2 j /(k+1)}, & 0 \leq u \ll 1, \\ \sum_{j \geq n} f_{j}^{-}|u|^{2 j /(l+1)}, & 0<-u \ll 1\end{cases}
$$

with $f_{m}^{+}=F_{m}^{+}\left((k+1) /\left(2 g_{k}^{+}(b)\right)\right)^{m /(k+1)}$ and $f_{n}^{-}=(-1)^{n} F_{n}^{-}\left((l+1) /\left(-2 g_{l}^{-}(b)\right)\right)^{n /(l+1)}$.

Proof. Let $H(y)=\int_{0}^{y} h(u) d u$ and make the transformation

$$
\begin{gathered}
u=\sqrt{G(x, b)}(\operatorname{sgn} x), \\
v=\sqrt{H(y)}(\operatorname{sgn} y),
\end{gathered}
$$

together with the scaling of the time $d \tau=(g(X(u)) h(Y(v)) / 2 u v) d t$ to system (1.13) so that (1.13) can be changed into

$$
\begin{gathered}
\dot{u}=v-K(v) F^{*}(u, a, b), \\
\dot{v}=-u,
\end{gathered}
$$

where $K(v)=v / h(Y(v))=(1 / \sqrt{2})+O(v) \in C^{\infty}, F^{*}(u, a, b)=F(X(u), a)$ and $X(u), Y(v)$ denote the inverse of the transformation (2.8). In fact, by (1.18) and similar to the proof of Lemma 2.1, we can obtain

$$
\begin{aligned}
X(u) & =\left\{\begin{array}{l}
X^{+}(u) \\
X^{-}(u)
\end{array}\right. \\
& = \begin{cases}\sum_{j \geq 1} x_{j}^{+} u^{2 j /(k+1)}, x_{1}^{+}=\left(\frac{k+1}{2 g_{k}^{+}(b)}\right)^{1 /(k+1)}, & 0 \leq u \ll 1, \\
\sum_{j \geq 1} x_{j}^{-}|u|^{2 j /(l+1)}, x_{1}^{-}=-\left(\frac{l+1}{-2 g_{l}^{-}(b)}\right)^{1 /(l+1)}, & 0<-u \ll 1 .\end{cases}
\end{aligned}
$$


For $0 \leq u \ll 1$, by (1.15) and (2.10), we have

$$
\begin{aligned}
F^{*}(u, a, b) & =F^{+}\left(X^{+}(u), a\right)=\sum_{j \geq m} F_{j}^{+}(a)\left(\sum_{i \geq 1} x_{i}^{+} u^{2 i /(k+1)}\right)^{j} \\
& =\sum_{j \geq m} f_{j}^{+} u^{2 j /(k+1)}
\end{aligned}
$$

where $f_{m}^{+}=F_{m}^{+}\left((k+1) / 2 g_{k}^{+}(b)\right)^{m /(k+1)}$. Similarly, for $0<-u \ll 1$, by (1.16) and (2.10), we can obtain

$$
F^{*}(u, a, b)=F^{-}\left(X^{-}(u), a\right)=\sum_{j \geq n} f_{j}^{-}|u|^{2 j /(l+1)},
$$

where $f_{n}^{-}=(-1)^{n} F_{n}^{-}\left((l+1) /-2 g_{l}^{-}(b)\right)^{n /(l+1)}$. This ends the proof. we have

For a relation between the function $F(x, a)$ in (1.13) and the function $F^{*}(u, a, b)$ in $(2.6)$

Lemma 2.3. Suppose (1.15) and (1.16) hold. Then we have

(i) $F^{-}(\alpha(x, b), a)-F^{+}(x, a)$ has the form (1.21) for $0 \leq x \ll 1$;

(ii) let $F_{0}(u, a, b)=F^{*}(-u, a, b)-F^{*}(u, a, b)$. Then $F_{0}$ can be expressed as

$$
F_{0}(u, a, b)=\sum_{j \geq 1} A_{j}(a, b) u^{2 k_{j} /(k+1)}, \quad \text { for } 0 \leq u \ll 1,
$$

where

$$
A_{1}(a, b)=B_{1}(a, b) N_{1}\left(x_{1}^{+}\right), \quad A_{j}(a, b)=B_{j}(a, b) N_{j}\left(x_{1}^{+}\right)+O\left(\left|B_{1}, B_{2}, \ldots, B_{j-1}\right|\right), \quad j \geq 2
$$

with $N_{j}(j \geq 1)$ are positive $C^{\infty}$ functions in $x_{1}^{+}$, and $k_{j}, j \geq 1$, are as appeared in (1.21).

Proof. By (1.15), (1.16), and Lemma 2.1, we can have

$$
\begin{aligned}
F^{-}(\alpha(x, b), a)-F^{+}(x, a) & =\sum_{s \geq n} F_{s}^{-}(a)\left[\sum_{i=1}^{p} x^{i r} \sum_{j \geq 0} \alpha_{i j} x^{j}\right]^{s}-\sum_{s \geq m} F_{s}^{+}(a) x^{s} \\
& =\sum_{s \geq n} F_{s}^{-}(a) x^{(q / p) s}\left[\sum_{i=0}^{p-1} x^{q i / p} \sum_{j \geq 0} \alpha_{i+1, j} x^{j}\right]^{s}-\sum_{s \geq m} F_{s}^{+}(a) x^{s} \\
& =\sum_{i=0}^{p-1} x^{(n+i) r} \sum_{j \geq 0} \bar{B}_{i j}(a, b) x^{j}-\sum_{s \geq m} F_{s}^{+}(a) x^{s}
\end{aligned}
$$


Since $r(n+\eta)$ is an integer, the above formula can be written as

$$
F^{-}(\alpha(x, b), a)-F^{+}(x, a)=\sum_{i=0, i \neq \eta}^{p-1} \sum_{j \geq 0} \bar{B}_{i j}(a, b) x^{(n+i) r+j}+\sum_{j \geq T} \widetilde{B}_{j}(a, b) x^{j},
$$

where $T=\min \{m, r(n+\eta)\}$. Then we express (2.16) with respect to the power of $x$ in ascending order, yielding the form of (1.21). In addition, for $k_{\tau}$ defined in Theorem 1.6 there must exist some integers $i_{\tau}$ and $j_{\tau}$ such that $k_{\tau}=\left(n+i_{\tau}\right) r+j_{\tau}$. Hence, we can obtain

$$
B_{\tau}(a, b)=\bar{B}_{i_{\tau} j_{\tau}} \quad \text { if } i_{\tau} \neq \eta, \quad B_{\tau}(a, b)=\widetilde{B}_{k_{\tau}} \quad \text { if } i_{\tau}=\eta
$$

This completes the proof of the conclusion (i).

Now we prove the conclusion (ii). For $u \geq 0$ sufficiently small, (2.8) and (2.10) imply that

$$
G^{+}\left(X^{+}(u), b\right)=G^{-}\left(X^{-}(-u), b\right)=u^{2} .
$$

Then noting that $G^{-}(\alpha(x, b), b)=G^{+}(x, b)$, we can get

$$
X^{-}(-u)=\alpha\left(X^{+}(u)\right)
$$

By (2.11), (2.12) and the above formula, we have for $u \geq 0$ sufficiently small

$$
\begin{aligned}
F_{0}(u, a, b) & =F^{*}(-u, a, b)-F^{*}(u, a, b)=\left[F^{-}\left(X^{-}(-u), a\right)-F^{+}\left(X^{+}(u), a\right)\right] \\
& =\left.\left[F^{-}(\alpha(x, b), a)-F^{+}(x, a)\right]\right|_{x=X^{+}(u)} .
\end{aligned}
$$

Let

$$
\varphi(\mu)=X^{+}\left(\mu^{(k+1) / 2}\right) .
$$

Combining (2.10) and (2.21) gives that

$$
\varphi(\mu)=\sum_{j \geq 1} x_{j}^{+} \mu^{j}=x_{1}^{+} \mu(1+O(\mu)) .
$$


Abstract and Applied Analysis

Substituting the above formula into (1.21) or (2.16) yields that

$$
\begin{aligned}
F^{-}(\alpha(x, b), a)-\left.F^{+}(x, a)\right|_{x=\varphi(\mu)}= & \sum_{j \geq 1} B_{j}(a, b)\left(x_{1}^{+}\right)^{k_{j}} \mu^{k_{j}}(1+O(\mu)) \\
= & \sum_{i=0, i \neq \eta}^{p-1} \sum_{j \geq 0} \bar{B}_{i j}(a, b)\left(\sum_{s \geq 1} x_{s}^{+} \mu^{s}\right)^{(n+i) r+j} \\
& +\sum_{j \geq T} \widetilde{B}_{j}(a, b)\left(\sum_{s \geq 1} x_{s}^{+} \mu^{s}\right)^{j}
\end{aligned}
$$

Note that

$$
\begin{aligned}
& \sum_{i=0, i \neq \eta}^{p-1} \sum_{j \geq 0} \bar{B}_{i j}(a, b)\left(\sum_{s \geq 1} x_{s}^{+} \mu^{s}\right)^{(n+i) r+j} \\
& =\sum_{i=0, i \neq \eta}^{p-1} \sum_{j \geq 0} \bar{B}_{i j}(a, b)\left(x_{1}^{+}\right)^{(n+i) r+j} \mu^{(n+i) r+j}\left(1+\sum_{s \geq 1} \frac{x_{s+1}^{+}}{x_{1}^{+}} u^{s}\right)^{(n+i) r+j} \\
& =\sum_{i=0, i \neq \eta}^{p-1} \sum_{j \geq 0} \bar{B}_{i j}(a, b)\left(x_{1}^{+}\right)^{(n+i) r+j} \mu^{(n+i) r+j}\left(1+\sum_{s \geq 1} \bar{x}_{s} \mu^{s}\right) \\
& =\sum_{i=0, i \neq \eta}^{p-1} \sum_{j \geq 0} B_{i j}^{*}(a, b) \mu^{(n+i) r+j}
\end{aligned}
$$

where

$$
B_{i 0}^{*}=\left(x_{1}^{+}\right)^{(n+i) r} \bar{B}_{i 0}, \quad B_{i j}^{*}=\left(x_{1}^{+}\right)^{(n+i) r+j} \bar{B}_{i j}+O\left(\left|\bar{B}_{i 0}, \bar{B}_{i 1}, \ldots, \bar{B}_{i, j-1}\right|\right), j \geq 1
$$

with $i=0, \ldots, p-1$ and $i \neq \eta$. Similarly,

$$
\sum_{j \geq T} \widetilde{B}_{j}(a, b)\left(\sum_{s \geq 1} x_{s}^{+} \mu^{s}\right)^{j}=\sum_{j \geq T} B_{j}^{*}(a, b) \mu^{j},
$$

where

$$
B_{T}^{*}=\left(x_{1}^{+}\right)^{T} \bar{B}_{T}, \quad B_{j}^{*}=\left(x_{1}^{+}\right)^{j} \bar{B}_{j}+O\left(\left|\bar{B}_{T}, \bar{B}_{T+1}, \ldots, \bar{B}_{j-1}\right|\right), \quad j \geq T+1 .
$$

Combining (2.23), (2.24), and (2.26), we can obtain

$$
F^{-}(\alpha(x, b), a)-\left.F^{+}(x, a)\right|_{x=\varphi(\mu)}=\sum_{i=0, i \neq \eta}^{p-1} \sum_{j \geq 0} B_{i j}^{*}(a, b) \mu^{(n+i) r+j}+\sum_{j \geq T} B_{j}^{*}(a, b) \mu^{j} .
$$


Similar to (1.21) and (2.16), the above formula can be rewritten as

$$
F^{-}(\alpha(x, b), a)-\left.F^{+}(x, a)\right|_{x=\varphi(\mu)}=\sum_{j \geq 1} A_{j}(a, b) \mu^{k_{j}},
$$

where from (2.17)

$$
A_{\tau}(a, b)=B_{i_{\tau} j_{\tau}}^{*} \quad \text { if } i_{\tau} \neq \eta, \quad A_{\tau}(a, b)=B_{k_{\tau}}^{*} \quad \text { if } i_{\tau}=\eta .
$$

From (2.25), (2.27), and the above equation, $A_{j}$ in (2.29) can be rewritten as the form of (2.14). Further, combining (2.17), (2.20), and (2.29) yields (2.13). Thus, the proof is ended.

Next, we establish the bifurcation function $d$ of system (1.13). For the purpose, let $u=r \cos \theta, v=r \sin \theta$. Then (2.6) can be translated into

$$
\frac{d r}{d \theta}=\frac{\cos \theta K(r \sin \theta) F^{*}(r \cos \theta, a, b)}{1-\sin \theta K(r \sin \theta) F^{*}(r \cos \theta, a, b) / r} \equiv R^{*}(\theta, r, a, b)
$$

In a similar way to the proof of Lemma 2.3 of [13], it is easy to prove that

$$
1-\frac{\sin \theta K(r \sin \theta) F^{*}(r \cos \theta, a, b)}{r}>0
$$

for $r>0$ small under $\left(\mathrm{H}_{1}\right)$ and $\left(\mathrm{H}_{2}\right)$, which shows that $R^{*}(\theta, r, a, b)$ in (2.31) is well defined. Introduce

$$
\beta=(k+1)(l+1), \quad \rho=r^{1 / \beta} .
$$

Then by (2.31) and (2.33), we can obtain

$$
\begin{aligned}
\frac{d \rho}{d \theta} & =\frac{\rho^{1-\beta}}{\beta} \times \frac{\cos \theta K\left(\rho^{\beta} \sin \theta\right) F^{*}\left(\rho^{\beta} \cos \theta, a, b\right)}{1-\sin \theta K\left(\rho^{\beta} \sin \theta\right) F^{*}\left(\rho^{\beta} \cos \theta, a, b\right) / \rho^{\beta}} \\
& =\frac{\rho}{\beta} \times \frac{\cos \theta K\left(\rho^{\beta} \sin \theta\right) F_{1}(\theta, \rho, a, b)}{1-\sin \theta K\left(\rho^{\beta} \sin \theta\right) F_{1}(\theta, \rho, a, b)} \equiv R(\theta, \rho, a, b),
\end{aligned}
$$

where $F_{1}(\theta, \rho, a, b)=\rho^{-\beta} F^{*}\left(\rho^{\beta} \cos \theta, a, b\right)$. Clearly, (2.34) is a $2 \pi$-periodic equation in $\theta$. By (2.7), we have

$$
F_{1}(\theta, \rho, a, b)= \begin{cases}\sum_{j \geq m} f_{j}^{+}(\cos \theta)^{2 j /(k+1)} \rho^{(2 j /(k+1)) \beta-\beta}, & \cos \theta \geq 0, \\ \sum_{j \geq n} f_{j}^{-}|\cos \theta|^{2 j /(l+1)} \rho^{(2 j /(l+1)) \beta-\beta}, & \cos \theta<0 .\end{cases}
$$




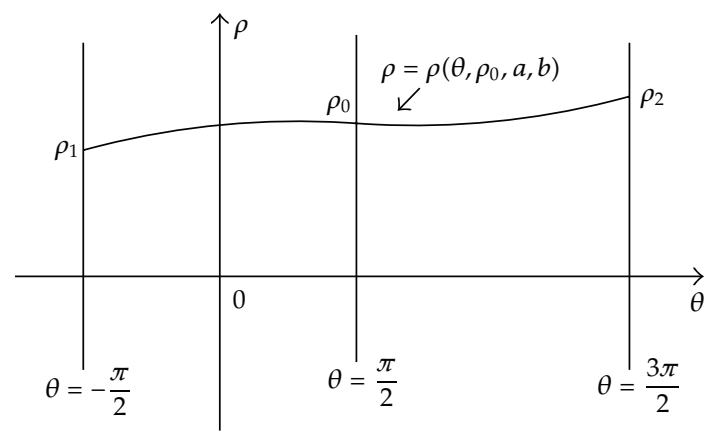

Figure 1

From (2.33) and (2.35), it is easy to see that $F_{1}$ is $C^{\infty}$ in $\rho$ for any given $\theta$. Let $\rho\left(\theta, \rho_{0}, a, b\right)$ denote the solution of (2.34) satisfying $\rho\left(\pi / 2, \rho_{0}, a, b\right)=\rho_{0}$. For convenience, let

$$
\rho\left(-\frac{\pi}{2}, \rho_{0}, a, b\right)=\rho_{1}, \quad \rho\left(\frac{3 \pi}{2}, \rho_{0}, a, b\right)=\rho_{2} .
$$

Define a function as follows:

$$
d\left(\rho_{0}, a, b\right)=\rho\left(-\frac{\pi}{2}, \rho_{0}, a, b\right)-\rho\left(\frac{3 \pi}{2}, \rho_{0}, a, b\right)=\rho_{1}-\rho_{2} .
$$

It is obvious that system (1.13), (2.6), (2.31), or (2.34) has a periodic orbit near the origin if and only if $d\left(\rho_{0}, a, b\right)$ in (2.37) has a zero in $\rho_{0}$ for $\rho_{0}>0$ sufficiently small. See Figure 1.

Hence, the function $d$ in (2.37) is called a displacement function or bifurcation function of system (1.13), (2.6), (2.31), or (2.34). About the bifurcation function $d$, we have.

Lemma 2.4. Let (1.15), (1.16), $\left(H_{1}\right)$, and $\left(H_{2}\right)$ hold. Then the bifurcation function $d$ in (2.37) has the form

$$
d\left(\rho_{0}, a, b\right)=\sum_{j \geq 1} C_{j}(a, b) \rho_{0}^{r_{j}}\left(1+d_{j}\left(\rho_{0}\right)\right)
$$

where $r_{j}=2(l+1) k_{j}-k l-k-l$ are positive integers satisfying $1 \leq r_{1}<r_{2}<\cdots$,

$$
\begin{gathered}
C_{1}(a, b)=B_{1}(a, b) N_{1}^{*}\left(f_{n}^{-}, f_{m}^{+}\right), \\
C_{j}(a, b)=B_{j}(a, b) N_{j}^{*}\left(f_{n}^{-}, f_{m}^{+}\right)+O\left(\left|B_{1}, B_{2}, \ldots, B_{j-1}\right|\right), \quad j \geq 2
\end{gathered}
$$

with $N_{j}^{*} \in C^{\infty}, N_{j}(0,0)>0$ for $j \geq 1$ and $d_{j}(j \geq 1)$ are $C^{\infty}$ functions in $\rho_{0}$ satisfying $d_{i}(0)=0$.

Proof. Denote $\bar{\rho}$ by $\bar{\rho}\left(\theta, \rho_{0}, a, b\right)=\rho\left(\pi-\theta, \rho_{0}, a, b\right)$. Then

$$
\bar{\rho}\left(\frac{\pi}{2}, \rho_{0}, a, b\right)=\rho\left(\frac{\pi}{2}, \rho_{0}, a, b\right), \quad \bar{\rho}\left(-\frac{\pi}{2}, \rho_{0}, a, b\right)=\rho\left(\frac{3 \pi}{2}, \rho_{0}, a, b\right)=\rho_{2}
$$


and it satisfies

$$
\frac{d \bar{\rho}}{d \theta}=-R(\pi-\theta, \rho, a, b)
$$

Let $\bar{R}(\theta, \rho, a, b)=-R(\pi-\theta, \rho, a, b)$. Then we can obtain that $\bar{\rho}\left(\theta, \rho_{0}, a, b\right)$ is a solution of the equation

$$
\frac{d \rho}{d \theta}=\bar{R}(\theta, \rho, a, b)
$$

satisfying $\bar{\rho}\left(\pi / 2, \rho_{0}, a, b\right)=\rho_{0}$. Let $\theta \in[-\pi / 2, \pi / 2]$. Then $\pi-\theta \in[\pi / 2,3 \pi / 2]$ and

$$
\begin{aligned}
& R(\theta, \rho, a, b)=R_{1}(\theta, a, b) \rho^{((2 m /(k+1))-1) \beta+1}(1+O(\rho)), \\
& \bar{R}(\theta, \rho, a, b)=\bar{R}_{1}(\theta, a, b) \rho^{((2 n /(l+1))-1) \beta+1}(1+O(\rho)),
\end{aligned}
$$

where

$$
\begin{aligned}
& R_{1}(\theta, a, b)= \begin{cases}\frac{K(0) f_{m}^{+}}{\beta}(\cos \theta)^{(2 m /(k+1))+1}, & m>\frac{k+1}{2}, \\
\frac{K(0) f_{m}^{+} \cos ^{2} \theta}{\beta\left(1-\sin \theta \cos \theta K(0) f_{m}^{+}\right)}, & m=\frac{k+1}{2},\end{cases} \\
& \bar{R}_{1}(\theta, a, b)= \begin{cases}-\frac{K(0) f_{n}^{-}}{\beta}(\cos \theta)^{(2 m /(l+1))+1}, & n>\frac{l+1}{2}, \\
-\frac{K(0) f_{n}^{-} \cos ^{2} \theta}{\beta\left(1-\sin \theta \cos \theta K(0) f_{n}^{-}\right)}, & n=\frac{l+1}{2} .\end{cases}
\end{aligned}
$$

Thus, by (2.43), (2.44) we have easily

$$
\begin{aligned}
& \rho\left(\theta, \rho_{0}, a, b\right)= \begin{cases}\rho_{0}+O\left(\rho_{0}^{((2 m /(k+1))-1) \beta+1}\right), & m>\frac{k+1}{2}, \\
\rho_{0} \exp \int_{\pi / 2}^{\theta} R_{1}(u, a, b) d u+O\left(\rho_{0}^{2}\right), & m=\frac{k+1}{2},\end{cases} \\
& \bar{R}\left(\theta, \rho\left(\theta, \rho_{0}, a, b\right), a, b\right)-\bar{R}\left(\theta, \bar{\rho}\left(\theta, \rho_{0}, a, b\right), a, b\right)=\left[\rho\left(\theta, \rho_{0}, a, b\right)-\bar{\rho}\left(\theta, \rho_{0}, a, b\right)\right] \Delta\left(\theta, \rho_{0}\right)
\end{aligned}
$$

by the mean value theorem, where $\Delta\left(\theta, \rho_{0}\right)=O\left(\rho_{0}^{((2 n /(l+1))-1) \beta}\right)$. Further by $(2.34)$ and $(2.42)$, we can obtain

$$
\begin{aligned}
& R(\theta, \rho, a, b)-\bar{R}(\theta, \rho, a, b) \\
& \quad=\frac{-\cos \theta K\left(\rho^{\beta} \sin \theta\right) \rho^{1-\beta}\left[F^{*}\left(-\rho^{\beta} \cos \theta, a, b\right)-F^{*}\left(\rho^{\beta} \cos \theta, a, b\right)\right]}{\beta\left[1-\sin \theta K\left(\rho^{\beta} \sin \theta\right) \rho^{-\beta} F^{*}\left(\rho^{\beta} \cos \theta, a, b\right)\right]\left[1-\sin \theta K\left(\rho^{\beta} \sin \theta\right) \rho^{-\beta} F^{*}\left(-\rho^{\beta} \cos \theta, a, b\right)\right]}
\end{aligned}
$$


which follows that by Lemma 2.3

$$
R(\theta, \rho, a, b)-\bar{R}(\theta, \rho, a, b)=-\cos \theta \rho^{1-\beta} F_{0}\left(\rho^{\beta} \cos \theta, a, b\right) S(\theta, \rho),
$$

where $S(\theta, \rho)$ is a $C^{\infty}$ function in $\rho$ with

$$
S(\theta, 0)= \begin{cases}\frac{K(0)}{\beta}, & m>\frac{k+1}{2}, n>\frac{l+1}{2}, \\ \frac{K(0)}{\beta\left(1-\sin \theta \cos \theta K(0) f_{m}^{+}\right)}, & m=\frac{k+1}{2}, n>\frac{l+1}{2}, \\ \frac{K(0)}{\beta\left(1-\sin \theta \cos \theta K(0) f_{n}^{-}\right)}, & m>\frac{k+1}{2}, n=\frac{l+1}{2}, \\ \frac{K(0)}{\beta\left(1-\sin \theta \cos \theta K(0) f_{m}^{+}\right)\left(1-\sin \theta \cos \theta K(0) f_{n}^{-}\right)}, & m=\frac{k+1}{2}, n=\frac{l+1}{2},\end{cases}
$$

which implies $S(\theta, 0)>0$ by the above discussion. Then it follows from (2.13), (2.45), and (2.48) that

$$
\begin{aligned}
& R\left(\theta, \rho\left(\theta, \rho_{0}, a, b\right), a, b\right)-\bar{R}\left(\theta, \rho\left(\theta, \rho_{0}, a, b\right), a, b\right) \\
& \quad=-\sum_{j \geq 1} A_{j}(a, b)(\cos \theta)^{\left(\left(2 k_{j} /(k+1)\right)+1\right)} \rho_{0}^{\left(2 k_{j} /(k+1)\right) \beta+1-\beta} q_{j}\left(\theta, \rho_{0}\right),
\end{aligned}
$$

where $q_{j}(j \geq 1)$ are $C^{\infty}$ functions in $\rho_{0}$ with

$$
q_{j}(\theta, 0)= \begin{cases}S(\theta, 0), & m>\frac{k+1}{2} \\ S(\theta, 0) \exp \left(\frac{2 k_{j}}{k+1} \beta \int_{\pi / 2}^{\theta} R_{1}(u, a, b) d u\right), & m=\frac{k+1}{2}\end{cases}
$$

which shows that $q_{j}(\theta, 0)>0$ for all $j \geq 1$. Since $\bar{\rho}(\theta, \rho, a, b)$ and $\rho(\theta, \rho, a, b)$ satisfy $(2.42)$ and (2.34), respectively, using (2.46) we have

$$
\frac{d}{d \theta}(\rho-\bar{\rho})=(\rho-\bar{\rho}) \Delta\left(\theta, \rho_{0}\right)+R(\theta, \rho, a, b)-\bar{R}(\theta, \rho, a, b),
$$

where $\bar{\rho}=\bar{\rho}\left(\theta, \rho_{0}, a, b\right)$ and $\rho=\rho\left(\theta, \rho_{0}, a, b\right)$. Then noting (2.40), applying the formula of variation of constants to the above equation yields

$$
\begin{aligned}
\rho\left(\theta, \rho_{0}, a, b\right)-\bar{\rho}\left(\theta, \rho_{0}, a, b\right)= & \int_{\pi / 2}^{\theta}\left(R(\delta, \rho(\delta, \rho, a, b), a, b)-\bar{R}\left(\delta, \rho\left(\delta, \rho_{0}, a, b\right), a, b\right)\right) \\
& \times \exp \left(\int_{\delta}^{\theta} \Delta\left(\tau, \rho_{0}\right) d \tau\right) d \delta .
\end{aligned}
$$


Inserting (2.50) into the above formula and taking $\theta=-\pi / 2$ give by (2.37) that

$$
\begin{aligned}
d\left(\rho_{0}, a, b\right)=\sum_{j \geq 1} & A_{j}(a, b) \rho_{0}^{\left(2 k_{j} /(k+1)\right) \beta+1-\beta} \int_{-\pi / 2}^{\pi / 2}(\cos \theta)^{\left(2 k_{j} /(k+1)\right)+1} q_{j}(\theta, 0) \\
& \times \exp \left(\int_{\delta}^{\theta} \Delta(\tau, 0) d \tau\right)\left(1+O\left(\rho_{0}\right)\right) d \theta
\end{aligned}
$$

which implies (2.38) by (2.33). Hence, The proof is completed.

In the following part, we verify Theorems 1.6 and 1.7 using the above lemmas.

Proof of Theorem 1.6. One can see that the conclusion (i) is true by Lemmas 2.1 and 2.3. Now, we prove the conclusion (ii). Obviously, it suffices to prove the following two points.

(1) There are at most $s$ limit cycles near the origin for all $(a, b)$ near $\left(a_{0}, b_{0}\right)$.

(2) There can appear $s$ limit cycles in any given neighborhood of the origin for some $(a, b)$ arbitrarily close to $\left(a_{0}, b_{0}\right)$.

In fact, noting that

$$
C_{s+1}\left(a_{0}, b_{0}\right)=B_{s+1}\left(a_{0}, b_{0}\right) N_{s+1}^{*} \neq 0 .
$$

By our assumption, we have from (2.38) and (2.55)

$$
\begin{aligned}
d\left(\rho_{0}, a, b\right) & =\sum_{j=1}^{s+1} C_{j}(a, b) \rho_{0}^{r_{j}}\left(1+d_{j}\left(\rho_{0}\right)\right)+\rho_{0}^{r_{s+2}} Q\left(\rho_{0}, a, b\right) \\
& =\sum_{j=1}^{s+1} C_{j}(a, b) \rho_{0}^{r_{j}}\left(1+\bar{d}_{j}\left(\rho_{0}\right)\right) \equiv D_{s},
\end{aligned}
$$

where $Q\left(\rho_{0}, a, b\right)=\sum_{j \geq s+2} C_{j}(a, b) \rho_{0}^{r_{j}-r_{s+1}}$,

$$
\bar{d}_{j}=d_{j}, \quad j=1, \ldots, s, \quad \bar{d}_{s+1}=d_{s+1}+\frac{Q\left(\rho_{0}, a, b\right)}{C_{s+1}(a, b)} \rho_{0}^{r_{s+2}-r_{s+1}},
$$

which implies $\bar{d}_{j}$ in (2.56) are $C^{\infty}$ in $\rho_{0}$ satisfying $\bar{d}_{j}(0)=0$ for $(a, b)$ sufficiently close to $\left(a_{0}, b_{0}\right)$.

We claim that $D_{s}$ in (2.56) has at most $s$ zeros in $\rho_{0}>0$ small for $\left|a-a_{0}\right|+\left|b-b_{0}\right| \ll 1$. We can proceed with the proof by induction on $s$.

First, when $s=1$, from (2.56), we can obtain

$$
\begin{aligned}
D_{1} & =\left(1+\bar{d}_{1}\left(\rho_{0}\right)\right) \rho_{0}^{r_{1}}\left[C_{1}(a, b)+C_{2}(a, b) \rho_{0}^{r_{2}-r_{1}}\left(1+\tilde{d}_{2}\left(\rho_{0}\right)\right)\right] \\
& =\left(1+\bar{d}_{1}\left(\rho_{0}\right)\right) \rho_{0}^{r_{1}} d_{1}\left(\rho_{0}, a, b\right) .
\end{aligned}
$$


Note that

$$
\frac{d d_{1}\left(\rho_{0}, a, b\right)}{d \rho_{0}}=C_{2}(a, b) \rho_{0}^{r_{2}-r_{1}-1}\left[\left(r_{2}-r_{1}\right)\left(1+\tilde{d}_{2}\left(\rho_{0}\right)\right)+\rho_{0} \tilde{d}_{2}^{\prime}\left(\rho_{0}\right)\right]
$$

which implies

$$
\frac{d d_{1}\left(\rho_{0}, a, b\right)}{d \rho_{0}} \neq 0
$$

for $0<\rho_{0} \ll 1$ and $(a, b)$ near $\left(a_{0}, b_{0}\right)$ since $C_{2}\left(a_{0}, b_{0}\right)=B_{2}\left(a_{0}, b_{0}\right) N_{2}^{*} \neq 0$ by (2.55). Hence, by Rolle's theorem, the conclusion (1) is true for $s=1$.

Assume that the conclusion (1) is true for $s=i$. That is by (2.56)

$$
D_{i}=\sum_{j=1}^{i+1} C_{j}(a, b) \rho_{0}^{r_{j}}\left(1+\bar{d}_{j}\left(\rho_{0}\right)\right)
$$

has at most $i$ zeros for $\rho_{0}>0$ and $\left|a-a_{0}\right|+\left|b-b_{0}\right|$ sufficiently small with $C_{i+1}\left(a_{0}, b_{0}\right) \neq 0$. Let us prove that the conclusion (1) is also true for $s=i+1$. Then from (2.56), we can obtain

$$
\begin{aligned}
D_{i+1} & =\sum_{j=1}^{i+2} C_{j}(a, b) \rho_{0}^{r_{j}}\left(1+\bar{d}_{j}\left(\rho_{0}\right)\right) \\
& =\rho_{0}^{r_{1}}\left(1+\bar{d}_{1}\left(\rho_{0}\right)\right) \sum_{j=1}^{i+2} C_{j}(a, b) \rho_{0}^{r_{j}-r_{1}} d_{j}^{*} \equiv \rho_{0}^{r_{1}}\left(1+\bar{d}_{1}\left(\rho_{0}\right)\right) \bar{D}_{i+1},
\end{aligned}
$$

where $d_{1}^{*}=1, d_{j}^{*}=\left(1+\bar{d}_{j}\left(\rho_{0}\right)\right) /\left(1+\bar{d}_{1}\left(\rho_{0}\right)\right)=1+O\left(\rho_{0}\right), j=2,3, \ldots, i+2$. Then,

$$
\begin{aligned}
\frac{d \bar{D}_{i+1}}{d \rho_{0}} & =\sum_{j=2}^{i+2}\left(r_{j}-r_{1}\right) C_{j}(a, b) \rho_{0}^{r_{j}-r_{1}-1}\left(d_{j}^{*}+\frac{\rho_{0}}{r_{j}-r_{1}} \frac{d d_{j}^{*}}{d \rho_{0}}\right) \\
& =\sum_{j=1}^{i+1} \bar{C}_{j}(a, b) \rho_{0}^{\tilde{r}_{j}}\left(1+\widehat{d}_{j}\left(\rho_{0}\right)\right)
\end{aligned}
$$

where $\bar{C}_{j}(a, b)=\left(r_{j+1}-r_{1}\right) C_{j+1}(a, b), \tilde{r}_{j}=r_{j+1}-r_{1}-1, j=1, \ldots, i+1$ satisfying $1 \leq \tilde{r}_{1}<$ $\cdots<\widetilde{r}_{i+1}$ and $\widehat{d}_{j}\left(\rho_{0}\right)$ is $C^{\infty}$ in $\rho_{0}$ with $\widehat{d}_{j}(0)=0$, which implies that $d \bar{D}_{i+1} / d \rho_{0}$ has at most $i$ zeros in $\rho_{0}>0$ small for $(a, b)$ near $\left(a_{0}, b_{0}\right)$ by induction assumption since $\bar{C}_{i+1}\left(a_{0}, b_{0}\right)=$ $\left(r_{i+2}-r_{1}\right) C_{i+2}\left(a_{0}, b_{0}\right) \neq 0$. Hence, by Rolle's theorem, it follows that $D_{i+1}$ in (2.62) has at most $i+1$ zeros in $0<\rho_{0} \ll 1$ for $(a, b)$ near $\left(a_{0}, b_{0}\right)$. This ends the proof of conclusion (1).

Now, we verify the conclusion (2). For simplicity, we can assume $(a, b)=\bar{a}=\left(\bar{a}_{1}\right.$, $\left.\bar{a}_{2}, \ldots, \bar{a}_{n_{1}}\right)$. Further, by (1.22), without loss of generality, suppose

$$
\operatorname{det} \frac{\partial\left(B_{1}, \ldots, B_{s}\right)}{\partial\left(\bar{a}_{1}, \ldots, \bar{a}_{s}\right)} \neq 0
$$


Then we can fix $\bar{a}_{j}=\bar{a}_{j 0}$ for $j=s+1, \ldots, n_{1}$ since $n_{1} \geq s$. Consider the equations

$$
B_{j}=B_{j}(\bar{a}), \quad j=1, \ldots, s .
$$

By the implicit function theorem, the equations have a unique set of solutions

$$
\bar{a}_{j}=\bar{a}_{j}^{*}\left(B_{1}, \ldots, B_{s}, \bar{a}_{s+1}, \ldots, \bar{a}_{n_{1}}\right), \quad j=1,2, \ldots, s
$$

for $\bar{a}$ near $\bar{a}_{0}$, which means $B_{j}(j=1, \ldots, s)$ can be taken as free parameters. Hence, by changing the sign of $B_{s}, B_{s-1}, \ldots, B_{1}$ in turn such that

$$
B_{j-1} B_{j}<0, \quad j=s+1, \ldots, 1,0<\left|B_{1}\right| \ll\left|B_{2}\right| \ll \cdots=\left|B_{s}\right| \ll 1,
$$

which follows that $C_{j}$ in (2.56) satisfy

$$
C_{j-1} C_{j}<0, \quad j=s+1, \ldots, 1,0<\left|C_{1}\right| \ll\left|C_{2}\right| \ll \cdots \ll\left|C_{s}\right| \ll 1 .
$$

This ensures that the bifurcation function $d$ has $s$ zeros in $\rho_{0}$ for some $(a, b)$ near $\left(a_{0}, b_{0}\right)$, which implies the conclusion (2). This ends the proof.

Proof of Theorem 1.7. From the proof of Theorem 1.6, it is easy to see $B_{j}(j=1, \ldots, s)$ can be taken as free parameters by $(1.22)$, which implies $C_{j}(j=1, \ldots, s)$ can also be taken as free parameters by the definition of $C_{j}$ in Lemma 2.4. From (1.23) and Lemma 2.4, we have

$$
d\left(\rho_{0}, a, b\right)=0 \quad \text { when } C_{j}=0, j=1, \ldots, s
$$

for $\left|a-a_{0}\right|+\left|b-b_{0}\right|$ sufficiently small since $B_{j}=0$ if and only if $C_{j}=0$, which means that the coefficients $C_{j}(j \geq s+1)$ in (2.38) satisfy

$$
C_{j}(a, b)=0, \quad j=s+1, s+2, \ldots \text { as long as } C_{1}=\cdots=C_{s}=0 .
$$

Since $C_{j}$ can be taken as free parameters, we can write

$$
C_{j}(a, b)=\sum_{i=1}^{s} C_{i} Q_{i j}(a, b)
$$

where $Q_{i j} \in C^{\infty}, j \geq s+1$. Inserting (2.71) into (2.38) gives that

$$
d\left(\rho_{0}, a, b\right)=\sum_{j=1}^{s} C_{j} \rho_{0}^{r_{j}}\left(1+e_{j}\left(\rho_{0}\right)\right)
$$

where

$$
e_{j}\left(\rho_{0}\right)=1+d_{j}\left(\rho_{0}\right)+\sum_{i \geq s+1} Q_{j i} \rho_{0}^{r_{i}-r_{j}}\left(1+d_{i}\left(\rho_{0}\right)\right) \quad \text { for } j=1, \ldots, s .
$$


We can easily prove $d$ in (2.72) has at most $s-1$ zeros for $(a, b)$ near $\left(a_{0}, b_{0}\right)$ by induction $s$ in a similar way to the above proof. Further, we can choose $C_{j}$ satisfying

$$
C_{j-1} C_{j}<0, \quad j=s, \ldots, 1,0<\left|C_{1}\right| \ll\left|C_{2}\right| \ll \cdots \ll\left|C_{s}\right| \ll 1,
$$

which ensures that $d$ in (2.72) has $s-1$ zeros for $(a, b)$ near $\left(a_{0}, b_{0}\right)$. The proof is finished.

From the proof of Theorems 1.6 and 1.7, we have immediately the following corollaries.

Corollary 2.5. Let (1.15), (1.16), $\left(H_{1}\right)$, and $\left(H_{2}\right)$ hold. If there exist a point $\left(a_{0}, b_{0}\right)$ and an integer $s \geq 1$ such that

$$
B_{j}\left(a_{0}, b_{0}\right)=0, \quad j=1,2, \ldots, s, \quad B_{s+1}\left(a_{0}, b_{0}\right)<0(\text { resp. },>0),
$$

then the origin is a stable (resp., unstable) focus of system (1.13) for $(a, b)=\left(a_{0}, b_{0}\right)$ and there has at most s limit cycles at the origin for all $(a, b)$ near $\left(a_{0}, b_{0}\right)$.

Corollary 2.6. Let (1.15), (1.16), $\left(H_{1}\right)$, and $\left(H_{2}\right)$ hold. If there exists $s \geq 1$ such that

$$
B_{j}=O\left(\left|B_{1}, B_{2}, \ldots, B_{s}\right|\right)
$$

for $j \geq s+1$, then for any given $N>0$ there exists a neighborhood $U$ of the origin such that for all $\left|B_{j}\right|<N, j=1, \ldots$, s system (1.13) has at most $s-1$ limits cycles in $U$.

Further, about system (1.13), we have the below two remarks in order.

Remark 2.7. If the function $h(y)$ in (1.13) has the form $h(y)=h_{1} y+O\left(y^{2}\right)$ with $h_{1}>0$, we also can obtain the same conclusions since this system is equivalent to the following:

$$
\begin{gathered}
\dot{x}=\frac{h(y)}{h_{1}}-\frac{F(x)}{h_{1}}, \\
\dot{y}=-\frac{g(x)}{h_{1}} .
\end{gathered}
$$

Remark 2.8. When vector parameter $a$ or $b$ is constant in (1.13), Theorems 1.6 and 1.7 remain true in this case.

\section{Applications}

In this section, we present some applications of our main results to systems of the form

$$
\begin{gathered}
\dot{x}=y-F(x, a), \\
\dot{y}=-g(x, b),
\end{gathered}
$$

where $F, g$ satisfy (1.15) and (1.16). 
Example 3.1. First consider system (3.1) with

$$
g(x, b)=\left\{\begin{array}{ll}
\sum_{j=0}^{k} b_{j} x^{j}, & x \geq 0, \\
-1, & x<0,
\end{array} \quad F(x, a)= \begin{cases}\sum_{j=1}^{n} a_{j}^{+} x^{j}, & x \geq 0, \\
\sum_{j=1}^{m} a_{j}^{-} x^{j}, & x<0,\end{cases}\right.
$$

where $b_{0}>0$ and $a=\left(a_{1}^{+}, \ldots, a_{n}^{+}, a_{1}^{-}, \ldots, a_{m}^{-}\right), b=\left(b_{0}, b_{1}, \ldots, b_{k}\right)$. By Lemma 2.1, it is clear that

$$
\alpha(x, b)=-\sum_{j=0}^{k} \frac{b_{j}}{j+1} x^{j+1}
$$

for $x \geq 0$ small. For any given $N>0$, denote $L(k, n, m)$ the cyclicity of system (3.1) near the origin for $|a|+|b| \leq N$. We study the cyclicity of system (3.1) under (3.2) near the origin with the following three cases.

Case 1. $m=1, k, m, n \geq 1$. In this case, we claim that $L(k, n, 1)=\max \{k+1, n\}-1$. In fact, by (3.2) and (3.3), we have

$$
\begin{aligned}
F^{-}(\alpha(x, b), a)-F^{+}(x, a) & =-\sum_{j=0}^{k} \frac{a_{1}^{-} b_{j}}{j+1} x^{j+1}-\sum_{j=1}^{n} a_{j}^{+} x^{j} \\
& =\sum_{j=1}^{\max \{k+1, n\}} B_{j}(a, b) x^{j}
\end{aligned}
$$

for $0 \leq x \ll 1$. From (3.4) it is easy to see that $F^{-}(\alpha(x, b), a) \equiv F^{+}(x, a)$ for $0 \leq x \ll 1$ if and only if $B_{j}=0$ for $j=1, \ldots, \max \{k+1, n\}$, which shows that $L(k, n, 1) \leq \max \{n, k+1\}-1$ by Corollary 2.6. Thus we only need to prove that $L(k, n, 1) \geq \max \{n, k+1\}-1$. For $n<k+1$, by (3.4), we can obtain

$$
B_{j}(a, b)=\frac{a_{1}^{-} b_{j-1}}{j}-a_{j}^{+}, \quad j=1, \ldots, n, \quad B_{j}(a, b)=\frac{a_{1}^{-} b_{j-1}}{j}, \quad j=n+1, \ldots, k+1 .
$$

Take $(a, b)=\left(a_{0}, \widetilde{b}_{0}\right)$ such that $a_{j 0}^{+}=\left(a_{10}^{-} b_{j-1,0}\right) / j, j=1, \ldots, n, b_{j 0}=0, j=n, \ldots, k$ and $a_{10}^{-} \neq 0$. Then by (3.5), we have

$$
B_{j}\left(a_{0}, b_{0}\right)=0, \quad j=1, \ldots, k+1,\left.\quad \operatorname{rank} \frac{\partial\left(B_{1}, \ldots, B_{n}, B_{n+1}, \ldots, B_{k+1}\right)}{\partial\left(a_{1}^{+}, \ldots, a_{n}^{+}, b_{n}, \ldots, b_{k}\right)}\right|_{\left(a_{0}, \tilde{b}_{0}\right)}=k+1
$$

which implies $k$ limit cycles can appear near the origin for $(a, b)$ near $\left(a_{0}, \tilde{b}_{0}\right)$. For $n \geq k+1$, we can discuss similarly. 
Case 2. $k=2, m=2, n \geq 1$. We will see that $L(2,2,1)=3, L(2,2,2)=L(2,2,3)=4, L(2,2,4)=$ $L(2,2,5)=5$, and $L(2,2, n)=n-1$ for $n \geq 6$.

In fact, combining (3.2) and (3.3) gives that

$$
\begin{aligned}
F^{-} & (\alpha(x, b), a)-F^{+}(x, a) \\
& =a_{1}^{-}\left(-b_{0} x-\frac{b_{1}}{2} x^{2}-\frac{b_{2}}{3} x^{3}\right)+a_{2}^{-}\left(-b_{0} x-\frac{b_{1}}{2} x^{2}-\frac{b_{2}}{3} x^{3}\right)^{2}-\sum_{j=1}^{n} a_{j}^{+} x^{j} \\
& =\sum_{j=1}^{\max \{6, n\}} B_{j}(a, b) x^{j} .
\end{aligned}
$$

As before, it is easy to get that $L(2,2, n)=n-1$ for $n \geq 6$. For $n \leq 5$, we can discuss system (3.1) case by case with respect to $n$.

For $n=1$, by (3.7) we can obtain

$$
\begin{gathered}
B_{1}(a, b)=-a_{1}^{-} b_{0}-a_{1}^{+}, \quad B_{2}(a, b)=-\frac{1}{2} a_{1}^{-} b_{1}+a_{2}^{-} b_{0}^{2}, \quad B_{3}(a, b)=-\frac{1}{3} a_{1}^{-} b_{2}+a_{2}^{-} b_{0} b_{1}, \\
B_{4}(a, b)=a_{2}^{-}\left(\frac{2}{3} b_{0} b_{2}+\frac{1}{4} b_{1}^{2}\right), \quad B_{5}(a, b)=\frac{1}{3} a_{2}^{-} b_{1} b_{2}, \quad B_{6}(a, b)=\frac{1}{9} a_{2}^{-} b_{2}^{2} .
\end{gathered}
$$

From (3.8), it is easy to see that $B_{5}=O\left(\left|B_{2}, B_{3}, B_{4}\right|\right), B_{6}=O\left(\left|B_{2}, B_{3}, B_{4}\right|\right)$. By Corollary 2.6, one can see that $L(2,2,1) \leq 3$. Further, from $(3.8)$ we can have $a_{0}=\left(a_{10}^{+}, a_{10}^{-}, a_{20}^{-}\right)$with

$$
a_{10}^{+}=-a_{10}^{-} b_{0}, \quad a_{20}^{-}=\frac{b_{1}}{2 b_{0}^{2}} a_{10}^{-}, \quad a_{10}^{-} \neq 0
$$

such that

$$
\begin{array}{r}
B_{1}\left(a_{0}, b\right)=B_{2}\left(a_{0}, b\right)=0, \quad B_{3}\left(a_{0}, b\right)=a_{10}^{-} \Delta_{1}(b), \\
B_{4}\left(a_{0}, b\right)=a_{10}^{-} \Delta_{2}(b), \\
B_{5}\left(a_{0}, b\right)=a_{10}^{-} \Delta_{3}(b), \quad B_{6}\left(a_{0}, b\right)=a_{10}^{-} \Delta_{4}(b),
\end{array}
$$

where

$$
\begin{gathered}
\Delta_{1}(b)=-\frac{b_{2}}{3}+\frac{b_{1}^{2}}{2 b_{0}}, \quad \Delta_{2}(b)=\frac{b_{1}}{b_{0}^{2}}\left(\frac{1}{3} b_{0} b_{2}+\frac{1}{8} b_{1}^{2}\right), \\
\Delta_{3}(b)=\frac{b_{1}^{2} b_{2}}{6 b_{0}^{2}}, \quad \Delta_{4}(b)=\frac{b_{1} b_{2}^{2}}{18 b_{0}^{2}} .
\end{gathered}
$$


Take $b=\widetilde{b}_{0}=\left(b_{00}, b_{10},\left(3 b_{10}^{2} / 2 b_{00}\right)\right)$ with $b_{00} \neq 0, b_{10} \neq 0$. Then by (3.11), we have

$$
\Delta_{1}\left(b_{0}\right)=0, \quad \Delta_{2}\left(b_{0}\right)=\frac{5 b_{10}^{3}}{8 b_{00}^{2}} \neq 0
$$

Note that

$$
\operatorname{det} \frac{\partial\left(B_{1}, B_{2}\right)}{\partial\left(a_{1}^{+}, a_{2}^{-}\right)}=\left|\begin{array}{cc}
-1 & 0 \\
0 & b_{0}^{2}
\end{array}\right|=-b_{0}^{2} \neq 0, \quad \frac{\partial \Delta_{1}}{\partial b_{2}}=-\frac{1}{3} \text {. }
$$

Then by Theorem 1.8, we know that system (3.1) has cyclicity 3 for $(a, b)$ sufficiently close to $\left(a_{0}, \tilde{b}_{0}\right)$. In other words, $L(2,2,1)=3$. Similarly, we have $L(2,2,2)=L(2,2,3)=4, L(2,2,4)=$ $L(2,2,5)=5$.

Case 3. $k=m=n, n=1,2,3$. We claim that $L(n, n, n)=3 n-2$ for $n=1,2,3$. In fact, for $k=m=n$, we have

$$
\begin{aligned}
F^{-}(\alpha(x, b), a)-F^{+}(x, a) & =\sum_{j=1}^{n} a_{j}^{-}\left(-\sum_{i=0}^{n} \frac{b_{i}}{i+1} x^{i+1}\right)^{j}-\sum_{j=1}^{n} a_{j}^{+} x^{j} \\
& =\sum_{j=1}^{n(n+1)} B_{j}(a, b) x^{j} .
\end{aligned}
$$

From Cases 1 and 2 , it is easy to see that $L(1,1,1)=1, L(2,2,2)=4$. For $k=m=n=3, B_{j}(a, b)$ in (3.14) can be written as

$$
\begin{gathered}
B_{1}(a, b)=-a_{1}^{-} b_{0}-a_{1}^{+}, \quad B_{2}(a, b)=-\frac{1}{2} a_{1}^{-} b_{1}+a_{2}^{-} b_{0}^{2}-a_{2}^{+}, \\
B_{3}(a, b)=-\frac{1}{3} a_{1}^{-} b_{2}+a_{2}^{-} b_{0} b_{1}-a_{3}^{-} b_{0}^{3}-a_{3}^{+}, \\
B_{4}(a, b)=-\frac{1}{4} a_{1}^{-} b_{3}+a_{2}^{-}\left(\frac{2}{3} b_{0} b_{2}+\frac{1}{4} b_{1}^{2}\right)-\frac{3}{2} a_{3}^{-} b_{0}^{2} b_{1}, \\
B_{5}(a, b)=a_{2}^{-}\left(\frac{1}{2} b_{0} b_{3}+\frac{1}{3} b_{1} b_{2}\right)-a_{3}^{-}\left(b_{0}^{2} b_{2}+\frac{3}{4} b_{0} b_{1}^{2}\right), \\
B_{6}(a, b)=a_{2}^{-}\left(\frac{1}{4} b_{1} b_{3}+\frac{1}{9} b_{2}^{2}\right)-a_{3}^{-}\left(b_{0} b_{1} b_{2}+\frac{3}{4} b_{0}^{2} b_{3}+\frac{1}{8} b_{1}^{3}\right), \\
B_{7}(a, b)=\frac{1}{6} a_{2}^{-} b_{2} b_{3}-a_{3}^{-}\left(\frac{3}{4} b_{0} b_{1} b_{3}+\frac{1}{3} b_{0} b_{2}^{2}+\frac{1}{4} b_{1}^{2} b_{2}\right), \\
B_{8}(a, b)=\frac{1}{16} a_{2}^{-} b_{3}^{2}-a_{3}^{-}\left(\frac{1}{2} b_{0} b_{2} b_{3}+\frac{3}{16} b_{1}^{2} b_{3}+\frac{1}{6} b_{1} b_{2}^{2}\right),
\end{gathered}
$$


Abstract and Applied Analysis

$$
\begin{array}{cc}
B_{9}(a, b)=-a_{3}^{-}\left(\frac{1}{4} b_{1} b_{2} b_{3}+\frac{3}{16} b_{0} b_{3}^{2}+\frac{1}{27} b_{2}^{3}\right), & B_{10}(a, b)=-a_{3}^{-}\left(\frac{3}{32} b_{1} b_{3}^{2}+\frac{1}{12} b_{2}^{2} b_{3}\right), \\
B_{11}(a, b)=-\frac{1}{15} a_{3}^{-} b_{2} b_{3}^{2}, \quad & B_{12}=-\frac{1}{64} a_{3}^{-} b_{3}^{3} .
\end{array}
$$

From (3.15), it is easy to prove that $B_{i}=O\left(\left|B_{4}, \ldots, B_{8}\right|\right), i=9, \ldots, 12$. Then by Corollary 2.6, we have $L(3,3,3) \leq 7$. In order to prove $L(3,3,3)=7$, let for convenience

$$
Z(b)=36 b_{0}^{2} b_{1} b_{3}-12 b_{0} b_{1}^{2} b_{2}-32 b_{0}^{2} b_{2}^{2}-9 b_{1}^{4}
$$

Then we can find $(a, b)=\left(a_{0}, \tilde{b}_{0}\right)$ with $Z\left(\tilde{b}_{0}\right) \neq 0$ and

$$
\begin{array}{cl}
a_{10}^{+}=-a_{10}^{-} b_{0}, \quad a_{20}^{+}=\frac{1}{2} a_{10}^{-} b_{1}-a_{20}^{-} b_{0}^{2}, & a_{30}^{+}=-\frac{1}{3} a_{10}^{-} b_{2}+a_{2}^{-} b_{0} b_{1}-a_{30}^{-} b_{0}^{3}, \\
a_{10}^{-} \neq 0, \quad a_{20}^{-}=\frac{3 a_{10}^{-} b_{3}\left(4 b_{0} b_{2}+3 b_{1}^{2}\right)}{Z(b)}, & a_{30}^{-}=\frac{2 a_{10}^{-} b_{3}\left(3 b_{0} b_{3}+2 b_{1} b_{2}\right)}{b_{0} Z(b)}
\end{array}
$$

for $b=\tilde{b}_{0}$ such that

$$
\begin{array}{ccc}
B_{1}\left(a_{0}, \tilde{b}_{0}\right)=\cdots=B_{5}\left(a_{0}, \tilde{b}_{0}\right)=0, \quad B_{6}\left(a_{0}, \tilde{b}_{0}\right)=a_{10}^{-} \Delta_{1}\left(\tilde{b}_{0}\right), \\
B_{7}\left(a_{0}, \tilde{b}_{0}\right)=a_{10}^{-} \Delta_{2}\left(\tilde{b}_{0}\right), & B_{8}\left(a_{0}, \tilde{b}_{0}\right)=a_{10}^{-} \Delta_{3}\left(\tilde{b}_{0}\right), & B_{9}\left(a_{0}, \tilde{b}_{0}\right)=a_{10}^{-} \Delta_{4}\left(\tilde{b}_{0}\right), \\
B_{10}\left(a_{0}, \tilde{b}_{0}\right)=a_{10}^{-} \Delta_{5}\left(\tilde{b}_{0}\right), & B_{11}\left(a_{0}, \tilde{b}_{0}\right)=a_{10}^{-} \Delta_{6}\left(\tilde{b}_{0}\right), & B_{12}\left(a_{0}, \tilde{b}_{0}\right)=a_{10}^{-} \Delta_{7}\left(\tilde{b}_{0}\right),
\end{array}
$$

where

$$
\begin{gathered}
\Delta_{1}(b)=Z_{1}\left(-\frac{9}{2} b_{0}^{3} b_{3}^{2}-6 b_{0}^{2} b_{1} b_{2} b_{3}+\frac{4}{3} b_{0}^{2} b_{2}^{3}+\frac{3}{2} b_{0} b_{1}^{2} b_{3}-3 b_{0} b_{1}^{2} b_{2}^{2}-\frac{1}{2} b_{1}^{4} b_{2}\right) \\
\Delta_{2}(b)=Z_{1}\left(-\frac{9}{2} b_{0}^{2} b_{1} b_{3}^{2}-3 b_{0} b_{1}^{2} b_{2} b_{3}^{2}-\frac{4}{3} b_{0} b_{1} b_{2}^{3}-b_{1}^{3} b_{2}^{2}\right) \\
\Delta_{3}(b)=Z_{1}\left(-\frac{9}{4} b_{0}^{2} b_{2} b_{3}^{2}-\frac{9}{16} b_{0} b_{1}^{2} b_{3}^{2}-3 b_{0} b_{1} b_{2}^{2} b_{3}-\frac{3}{4} b_{1}^{2} b_{2} b_{3}-\frac{2}{3} b_{1}^{2} b_{2}^{3}\right) \\
\Delta_{4}(b)=Z_{1}\left(-\frac{9}{8} b_{0}^{2} b_{3}^{2}-\frac{9}{4} b_{0} b_{1} b_{2} b_{3}^{2}-\frac{2}{9} b_{0} b_{2}^{3} b_{3}-b_{1}^{2} b_{2}^{2} b_{3}-\frac{4}{27} b_{1} b_{2}^{4}\right) \\
\Delta_{5}(b)=Z_{1}\left(-\frac{9}{16} b_{0} b_{1} b_{3}^{2}-\frac{1}{2} b_{0} b_{2}^{2} b_{3}^{2}-\frac{3}{8} b_{1}^{2} b_{2} b_{3}^{2}-\frac{1}{3} b_{1} b_{2}^{3} b_{3}\right) \\
\Delta_{6}(b)=Z_{1}\left(-\frac{3}{8} b_{2} b_{3}^{3}-\frac{1}{4} b_{1} b_{2}^{2} b_{3}^{2}\right), \quad \Delta_{7}(b)=Z_{1}\left(-\frac{3}{32} b_{0} b_{3}^{4}-\frac{1}{16} b_{1} b_{2} b_{3}^{2}\right)
\end{gathered}
$$


with $Z_{1}=b_{3} / b_{0} Z$. Let's choose $\tilde{b}_{0}=\left(b_{00}, 0,(3 / 2) b_{00}^{1 / 3} b_{30}^{2 / 3}, b_{30}\right)$ with $b_{00}>0, b_{30} \neq 0$. Obviously, $Z\left(\tilde{b}_{0}\right) \neq 0$. Then by (3.19), we have

$$
\Delta_{1}\left(\tilde{b}_{0}\right)=\Delta_{2}\left(\tilde{b}_{0}\right)=0, \quad \Delta_{3}\left(\tilde{b}_{0}\right)=\frac{3 b_{30}^{7 / 3}}{64 b_{00}^{4 / 3}} \neq 0 .
$$

Note that

$$
\begin{gathered}
\left.\operatorname{det} \frac{\partial\left(B_{1}, B_{2}, B_{3}, B_{4}, B_{5}\right)}{\partial\left(a_{1}^{+}, a_{2}^{+}, a_{3}^{+}, a_{2}^{-}, a_{3}^{-}\right)}\right|_{\left(a_{0}, \tilde{b}_{0}\right)}=\frac{9}{8} b_{00}^{8 / 3} b_{30}^{4 / 3} \neq 0, \\
\left.\operatorname{det} \frac{\partial\left(\Delta_{1}, \Delta_{2}\right)}{\partial\left(b_{1}, b_{2}\right)}\right|_{\tilde{b}_{0}}=\frac{81}{2} b_{00}^{14 / 3} b_{30}^{10 / 3} \neq 0 .
\end{gathered}
$$

Then by Theorem 1.8, it is clear that 7 limit cycles can appear near the origin for $(a, b)$ sufficiently close $\left(a_{0}, \tilde{b}_{0}\right)$. Then we have $L(3,3,3)=7$.

Example 3.2. Consider system (3.1) with

$$
g(x, b)=\left\{\begin{array}{ll}
x^{2}, \quad x \geq 0, \\
-1+b_{1} x+b_{2} x^{2}, \quad x<0,
\end{array} \quad F(x, a)= \begin{cases}\sum_{j=2}^{n} a_{j}^{+} x^{j}, & x \geq 0, \\
\sum_{j=1}^{m} a_{j}^{-} x^{j}, & x<0 .\end{cases}\right.
$$

Clearly, the origin is a center or focus by Theorem 1.2. Denote $H(m, n)$ the cyclicity of system (3.1) near the origin. By (3.22), we can obtain

$$
G(x, b)= \begin{cases}\frac{x^{3}}{3}, & x \geq 0, \\ -x+\frac{b_{1}}{2} x^{2}+\frac{b_{2}}{3} x^{3}, & x<0 .\end{cases}
$$

Using Lemma 2.1 and (3.23), for $x \geq 0$ small, we can solve from the equation $G^{-}(\alpha(x, b), b)=$ $G^{+}(x, b)$

$$
\begin{aligned}
\alpha(x, b)=\sum_{j \geq 1} \alpha_{j} x^{3 j}= & -\frac{1}{3} x^{3}+\frac{1}{18} b_{1} x^{6}-\left(\frac{b_{1}^{2}}{54}+\frac{b_{2}}{81}\right) x^{9}+\left(\frac{5}{648} b_{1}^{3}+\frac{5}{486} b_{1} b_{2}\right) x^{12} \\
& -\left(\frac{7}{1944} b_{1}^{4}+\frac{7}{972} b_{1}^{2} b_{2}+\frac{1}{729} b_{2}^{2}\right) x^{15} \\
& +\left(\frac{7}{3888} b_{1}^{5}+\frac{7}{1458} b_{1}^{3} b_{2}+\frac{14}{6561} b_{1} b_{2}^{2}\right) x^{18}+O\left(x^{21}\right) .
\end{aligned}
$$


Take $m=4, n=5$. Combining (3.22) and (3.24), we can obtain

$$
F^{-}(\alpha(x, b), a)-F^{+}(x, a)=\sum_{j=1}^{5} B_{j}(a, b) x^{j+1}+\sum_{j \geq 6} B_{j}(a, b) x^{3(j-3)},
$$

where

$$
\begin{gathered}
B_{1}(a, b)=-a_{2}^{+}, \quad B_{2}(a, b)=-a_{3}^{+}-\frac{1}{3} a_{1}^{-}, \quad B_{3}(a, b)=-a_{4}^{+}, \quad B_{4}=-a_{5}^{+}, \\
B_{5}(a, b)=\frac{1}{18} a_{1}^{-} b_{1}+\frac{1}{9} a_{2}^{-}, \quad B_{6}(a, b)=a_{1}^{-}\left(-\frac{1}{54} b_{1}^{2}-\frac{1}{81} b_{2}\right)-\frac{1}{27} a_{2}^{-} b_{1}-\frac{1}{27} a_{3}^{-}, \\
B_{7}(a, b)=a_{1}^{-}\left(\frac{5}{648} b_{1}^{3}+\frac{5}{486} b_{1} b_{2}\right)+a_{2}^{-}\left(\frac{5}{324} b_{1}^{2}+\frac{2}{243} b_{2}\right)+\frac{1}{54} a_{3}^{-} b_{1}+\frac{1}{81} a_{4}^{-}, \\
B_{8}(a, b)=a_{1}^{-}\left(-\frac{1}{1944} b_{1}^{4}-\frac{1}{972} b_{1}^{2} b_{2}-\frac{1}{729} b_{2}^{2}\right)+a_{2}^{-}\left(-\frac{7}{972} b_{1}^{3}-\frac{2}{243} b_{1} b_{2}\right) \\
\quad-a_{3}^{-}\left(\frac{1}{108} b_{1}^{2}+\frac{1}{243} b_{2}\right)-\frac{2}{243} a_{4}^{-} b_{1}, \\
B_{9}(a, b)=a_{1}^{-}\left(\frac{7}{3888} b_{1}^{5}+\frac{7}{1458} b_{1}^{3} b_{2}+\frac{14}{6561} b_{1} b_{2}^{2}\right)+a_{2}^{-}\left(\frac{1}{648} b_{1}^{4}+\frac{5}{2187} b_{1}^{2} b_{2}+\frac{7}{6561} b_{2}^{2}\right) \\
+a_{3}^{-}\left(\frac{7}{1458} b_{1}^{3}+\frac{7}{1458} b_{1} b_{2}\right)+a_{4}^{-}\left(\frac{7}{1458} b_{1}^{2}+\frac{4}{2187} b_{2}\right),
\end{gathered}
$$

which implies that $B_{j}=O\left(\left|B_{1}, \ldots, B_{9}\right|\right), j \geq 10$. Then we can obtain $H(4,5) \leq 8$ by Corollary 2.6. Now we prove that $H(4,5)=8$. Suppose $a=\left(a_{2}^{+}, \ldots, a_{5}^{+}, a_{1}^{-}, \ldots, a_{4}^{-}\right)$. Then we can find

$$
a_{0}=\left(\bar{a}_{20}^{+}, \ldots, \bar{a}_{50}^{+}, \bar{a}_{10}^{-}, \ldots, \bar{a}_{40}\right),
$$

where

$$
a_{20}^{+}=a_{40}^{+}=a_{50}^{+}=a_{40}^{-}=0, \quad a_{30}^{+}=-\frac{1}{3} a_{10}^{-}, \quad a_{20}^{-}=-\frac{1}{2} a_{10}^{-} b_{1}, \quad a_{30}^{-}=-\frac{1}{3} a_{10}^{-} b_{2}, \quad a_{10}^{-} \neq 0,
$$

such that

$$
\begin{gathered}
B_{1}\left(a_{0}, b\right)=B_{2}\left(a_{0}, b\right)=B_{3}\left(a_{0}, b\right)=B_{4}\left(a_{0}, b\right)=B_{5}\left(a_{0}, b\right)=B_{6}\left(a_{0}, b\right)=B_{7}\left(a_{0}, b\right)=0, \\
B_{8}\left(a_{0}, b\right)=\frac{1}{162} a_{1}^{-} \Delta_{0}, \quad B_{9}\left(a_{0}, b\right)=\frac{a_{1}^{-}}{162} \Delta_{1},
\end{gathered}
$$


where

$$
\Delta_{0}=b_{1}^{2}\left(\frac{1}{2} b_{1}^{2}+b_{2}\right), \quad \Delta_{1}=b_{1}^{3}\left(\frac{5185}{31096} b_{1}^{2}+\frac{1}{3} b_{2}\right)
$$

Solving the equation $\Delta_{0}=0$ gives $b=\left(b_{10},-(1 / 2) b_{10}^{2}\right) \equiv b_{0}, b_{10} \neq 0$. Inserting $b_{0}$ into (3.30) gives

$$
\Delta_{1}\left(b_{0}\right)=\frac{5185}{186576} b_{10}^{5} \neq 0
$$

Further, by (3.26) and (3.30), we have

$$
\begin{aligned}
\frac{\partial\left(B_{1}, B_{2}, B_{3}, B_{4}, B_{5}, B_{6}, B_{7}\right)}{\partial\left(a_{2}^{+}, a_{3}^{+}, a_{4}^{+}, a_{5}^{+}, a_{2}^{-}, a_{3}^{-}, a_{4}^{-}\right)}= & \left(\begin{array}{ccccccc}
-1 & 0 & 0 & 0 & 0 & 0 & 0 \\
0 & -1 & 0 & 0 & 0 & 0 & 0 \\
0 & 0 & -1 & 0 & 0 & 0 & 0 \\
0 & 0 & 0 & -1 & 0 & 0 & 0 \\
0 & 0 & 0 & 0 & -\frac{1}{9} & 0 & 0 \\
0 & 0 & 0 & 0 & -\frac{b_{1}}{27} & -\frac{1}{27} & 0 \\
0 & 0 & 0 & 0 & \frac{5 b_{1}^{2}}{324}+\frac{2 b_{2}}{243} & \frac{b_{1}}{54} & \frac{1}{81}
\end{array}\right), \\
& \frac{\partial \Delta_{0}}{\partial b_{2}}\left(b_{0}\right)=b_{10}^{2} \neq 0 .
\end{aligned}
$$

Hence, by the conclusion (i) of Theorem 1.8, it is easy to see that there exists cyclicity 8 when $(a, b)$ near $\left(a_{0}, b_{0}\right)$, which implies $H(4,5)=8$.

Remark 3.3. In a similar way, for $1 \leq m \leq 4,2 \leq n \leq 5$, we can obtain $H(m, n)=m+n-1$ for $n \neq 2, H(m, n)=m+n-2$ for $n=2, m \neq 2$ and $H(2,2)=3$.

\section{References}

[1] N. Glade, L. Forest, and J. Demongeot, "Liénard systems and potential-Hamiltonian decomposition. III. Applications," Comptes Rendus Mathématique, vol. 344, no. 4, pp. 253-258, 2007.

[2] J. Llibre, "A survey on the limit cycles of the generalization polynomial Linard differential equations. Mathematical models in engineering, biology and medicine," in Proceedings of the AIP International Conference on Boundary Value Problems: Mathematical Models in Engineering, Biology and Medicine, vol. 1124, pp. 224-233, The American Institute of Physics, Melville, NY, USA, 2009.

[3] M. Han, H. Zang, and T. Zhang, "A new proof to Bautin's theorem," Chaos, Solitons and Fractals, vol. 31, no. 1, pp. 218-223, 2007.

[4] L. A. Cherkas, "Conditions for the center for certain equations of the form $y y^{\prime}=P(x)+Q(x) y+$ $R(x) y^{2}, "$ Difference Equations, vol. 8, pp. 1104-1107, 1972.

[5] A. Gasull, "Differential equations that can be transformed into equations of Liénard type," 1989.

[6] J. Giné and J. Llibre, "Weierstrass integrability in Liénard differential systems," Journal of Mathematical Analysis and Applications, vol. 377, no. 1, pp. 362-369, 2011.

[7] L. A. Cherkas, "Conditions for a Lienard equation to have a center," Differential Equations, vol. 12, pp. 201-206, 1976. 
[8] L. A. Cherkas and V. G. Romanovski, "The center conditions for a Liénard system," Computers and Mathematics with Applications, vol. 52, no. 3-4, pp. 363-374, 2006.

[9] A. Gasull and J. Torregrosa, "Center problem for several differential equations via Cherkas' method," Journal of Mathematical Analysis and Applications, vol. 228, no. 2, pp. 322-343, 1998.

[10] M. A. Han, "Criteria for the existence of closed orbits of autonomous systems in the plane," Journal of Nanjing University, vol. 21, no. 2, pp. 233-244, 1985 (Chinese).

[11] M. Han, "Liapunov constants and Hopf cyclicity of Liénard systems," Annals of Differential Equations, vol. 15, no. 2, pp. 113-126, 1999.

[12] M. Han and W. Zhang, "On Hopf bifurcation in non-smooth planar systems," Journal of Differential Equations, vol. 248, no. 9, pp. 2399-2416, 2010.

[13] X. Liu and M. Han, "Hopf bifurcation for nonsmooth Liénard systems," International Journal of Bifurcation and Chaos in Applied Sciences and Engineering, vol. 19, no. 7, pp. 2401-2415, 2009.

[14] J. Llibre and C. Valls, "On the number of limit cycles of a class of polynomial differential systems," Proceedings of the Royal Society, vol. 468, no. 2144, pp. 2347-2360, 2012.

[15] Y. Q. Ye, S. L. Cai, L. S. Chen et al., Theory of Limit Cycles, vol. 66 of Translations of Mathematical Monographs, American Mathematical Society, Providence, RI, USA, 2nd edition, 1986.

[16] M. Han, J. Yang, and P. Yu, "Hopf bifurcations for near-Hamiltonian systems," International Journal of Bifurcation and Chaos in Applied Sciences and Engineering, vol. 19, no. 12, pp. 4117-4130, 2009.

[17] M. Han, C. Li, and J. Li, "Limit cycles of planar polynomial vector fields," Scholarpedia, vol. 5, no. 8, article 9648, 2010. 


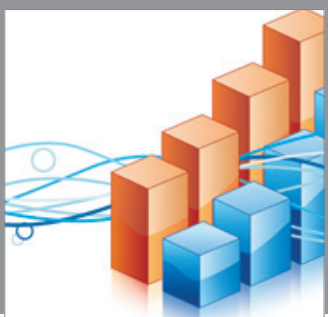

Advances in

Operations Research

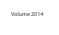

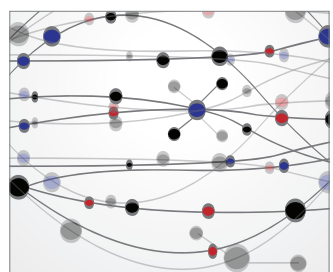

\section{The Scientific} World Journal
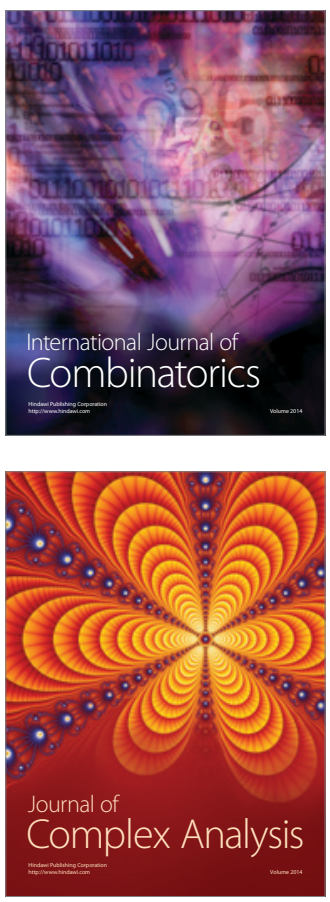

International Journal of

Mathematics and

Mathematical

Sciences
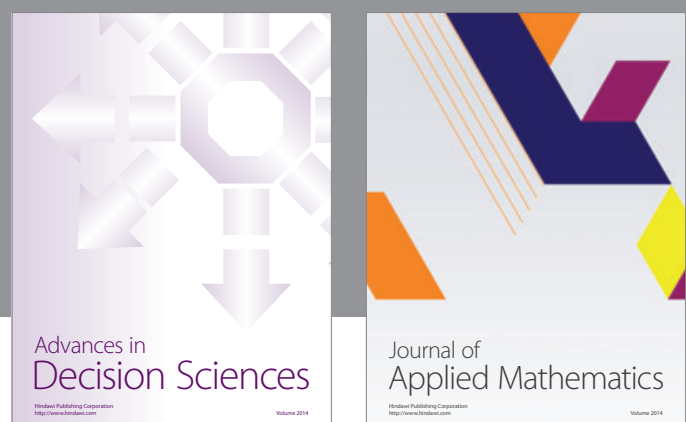

Journal of

Applied Mathematics
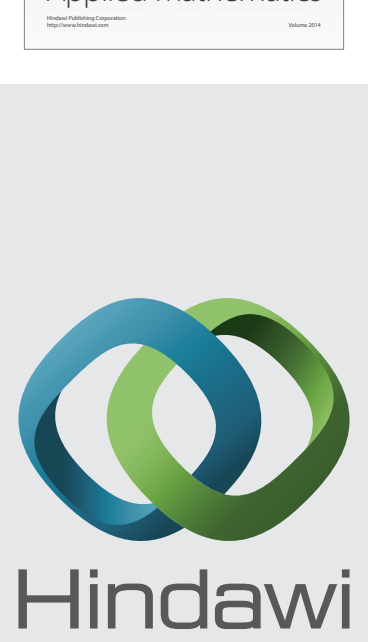

Submit your manuscripts at http://www.hindawi.com
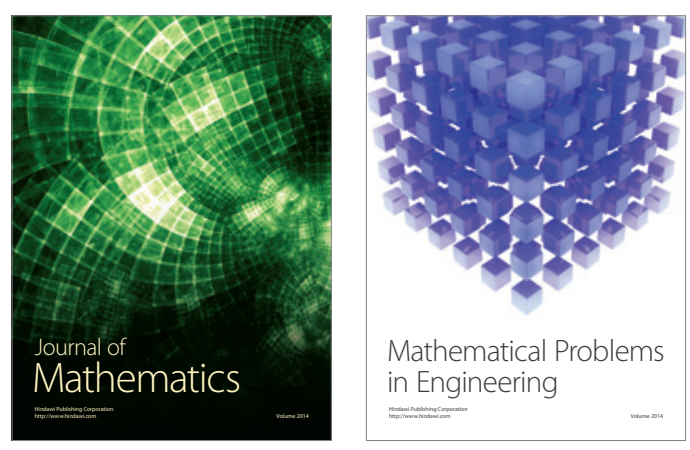

Mathematical Problems in Engineering
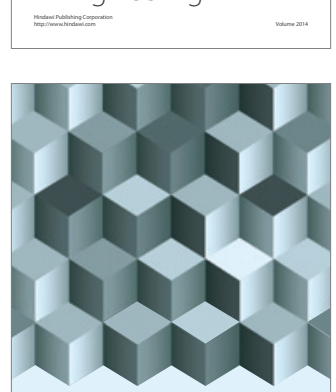

Journal of

Function Spaces
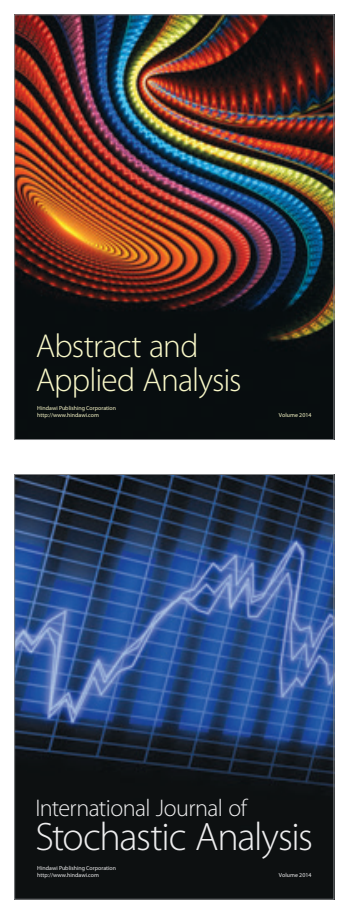

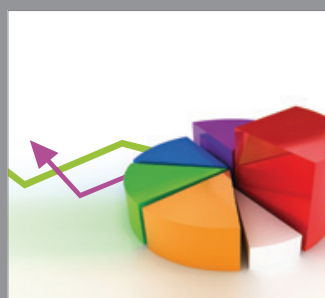

ournal of

Probability and Statistics

Promensencen
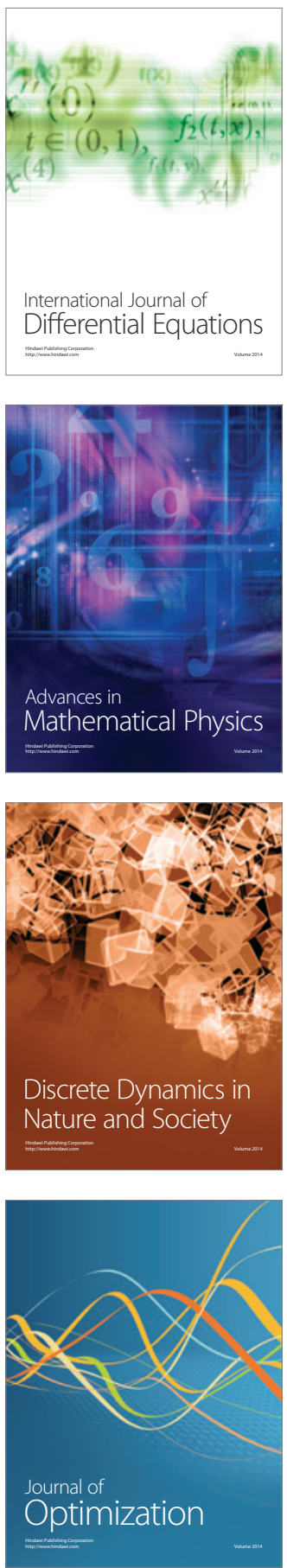